\title{
SUPERCONTINUUM GENERATION IN YAG AND SAPPHIRE WITH PICOSECOND LASER PULSES
}

\author{
I. Gražulevičiūtė, M. Skeivytė, E. Keblytė, J. Galinis, G. Tamošauskas, and A. Dubietis \\ Department of Quantum Electronics, Vilnius University, Sauletekio 9, LT-10222 Vilnius, Lithuania \\ E-mail: audrius.dubietis@ff.vu.lt
}

Received 10 March 2015; revised 10 April 2015; accepted 15 June 2015

\begin{abstract}
We report on experimental investigation of supercontinuum generation in YAG and sapphire crystals using $1.1 \mathrm{ps,} 1055 \mathrm{~nm}$ laser pulses. Under loose focusing conditions a stable single filament is generated without the onset of optical damage within the input power range of 6 to $10 P_{\mathrm{cr}}$, as verified from the near and far field measurements, and which gives rise to stable and reproducible supercontinuum spanning from $460 \mathrm{~nm}$ to $1.4 \mu \mathrm{m}$ in YAG, and from $410 \mathrm{~nm}$ to $1.3 \mu \mathrm{m}$ in sapphire. Permanent material modification, which develops under multiple laser shot exposure, is identified as the main limiting factor, which deteriorates the intensity distribution of the filament and dramatically alters the supercontinuum spectra.
\end{abstract}

Keywords: self-focusing and filamentation, supercontinuum generation, permanent material modification

PACS: 42.65.Jx, 42.65.Re

\section{Introduction}

White light continuum or supercontinuum (SC) generation in bulk dielectric media is a simple and very efficient method for production of broadband radiation with excellent spatial and temporal coherence properties at various parts of the optical spectrum and finds numerous applications in ultrafast pump-probe spectroscopy and contemporary nonlinear optics. During the last decades, SC generation was intensively studied in a variety of wide-bandgap solid-state media with femtosecond laser pulses, using amplified Ti:sapphire lasers as predominant pump sources, see e. g. [1-9]. More recently, efficient SC generation was also reported with ultrashort near [6, 10-13] and mid 14. 15 infrared pulses from the optical parametric amplifiers, resulting in ultrabroadband, several octavespanning SC spectra. These experiments demonstrate that filamentation of femtosecond laser pulses in wide bandgap dielectric media could be safely performed by choosing proper, low numerical aperture focusing conditions, under which filamentation and SC generation take place without the onset of the optical damage.

On the other hand, supercontinuum generation with picosecond laser pulses has been performed so far in liquids and gases, where no permanent optical dam- age occurs [16]. However, supercontinuum generation with picosecond laser pulses in solid state media is always considered as a difficult task and poses stringent requirements for the nonlinear medium, such as high optical damage threshold, polishing and crystalline quality. Only recently stable SC generation in YAG and other laser host crystals has been studied with Ti:sapphire laser pulses of variable duration accessed via either spectral narrowing or pulse chirping [17, 18] and, somewhat later, with genuine sub-picosecond and picosecond laser pulses from a newly developed Yb:YAG Innoslab amplifier system [19, 20]. The latter works demonstrated its straightforward application to ultrabroadband table-top optical parametric chirpedpulse amplification (OPCPA)-based systems, where picosecond SC seeding markedly simplifies the overall setup, excluding the need of employing an optically synchronized broadband laser oscillator source.

Despite these efforts, SC generation in solid state media with picosecond laser pulses is still a poorly investigated topic and faces a number of challenges concerning optical degradation of the material and, eventually, catastrophic optical damage. In this paper we present a detailed study of SC generation in YAG and sapphire crystals using 1.1 ps pump pulses with a centre wavelength of $1055 \mathrm{~nm}$. We identify the 
operating conditions for stable and broadband SC generation and reveal that permanent material modification, which develops under multiple laser shot exposure, is the main limiting factor, which should be carefully accounted for as far as further applications are concerned.

\section{Experimental setup and materials}

The experiment was performed using an amplified Nd:glass laser system (Twinkle, Light Conversion Ltd.), which delivers $1.1 \mathrm{ps}, 1055 \mathrm{~nm}$ pulses with energy up to $5 \mathrm{~mJ}$ at $10 \mathrm{~Hz}$ repetition rate. The laser output beam was suitably attenuated, reduced in size, spatially filtered and thereafter loosely focused by an $f=+225 \mathrm{~mm}$ lens into a $100 \mu \mathrm{m}$ FWHM spot size at the input face of the nonlinear medium located at the focal plane of the lens. The input energy was varied by means of a neutral filter with variable density. The input energy range was chosen so as to avoid single-shot optical damage of the nonlinear medium. That was verified by monitoring the near-field intensity distribution of the output beam (filament) using a CCD camera (COHU with a Spiricon frame grabber) and the far-field SC pattern in the visible spectral range using a digital photocamera. High dynamic range spectral measurements were performed using two mutually calibrated fiber spectrometers (AvaSpec-2048, AvaSpec-NIR256-2.5, both from Avantes), altogether covering the $0.3-2.5 \mu \mathrm{m}$ effective spectral range and operating in a single-shot regime. The SC spectra were composed of two laser shots and corrected according to sensitivity functions of the spectrometers and transmission of the colour filters, and a partially reflecting mirror for the suppression of the pump wavelength used in the measurement.

The nonlinear media used in the experiment were $15 \mathrm{~mm}$ long sapphire and YAG crystals. As the sapphire crystal is birefringent, its orientation was set so as the input beam propagated along the optical axis in order to avoid polarization dependent effects. Both crystals provide a useful combination of high nonlinearity and high optical damage threshold, and their relevant linear and nonlinear properties are listed in Table.

Table. Relevant parameters of YAG and sapphire: $n_{0}$ and $n_{2}$ are linear refractive indexes, respectively, $P_{\mathrm{cr}}$ is the critical power for self-focusing and $F_{\mathrm{th}}$ is the damage threshold. Data from [21-23].

\begin{tabular}{|c|c|c|c|c|}
\hline Crystal & $n_{0}$ & $n_{2}$ & $P_{\mathrm{cr}}$ & $F_{\text {th }}$ \\
\hline & & $10^{-16} \mathrm{~cm}^{2} / \mathrm{W}$ & MW & $\mathrm{J} / \mathrm{cm}^{2}$ \\
\hline YAG & 1.83 & 6.5 & 1.4 & 7.5 \\
\hline Sapphire & 1.75 & 3.3 & 2.9 & $5-8.8$ \\
\hline
\end{tabular}

\section{Supercontinuum generation in YAG and sapphire}

The spectral broadening in YAG was detected with the input pulse energy of $11 \mu \mathrm{J}$, that corresponds to $7.1 P_{\text {cr? }}$ where $P_{\mathrm{cr}}=0.15 \lambda^{2} / n_{0} n_{2}$ is the critical power for selffocusing. Figure 1 shows the examples of the SC spectra generated in YAG, as measured by increasing the input-pulse energy from 12 to $22 \mu \mathrm{J}$. With the input pulse energy of $12 \mu \mathrm{J}$ the SC spectrum extends from $460 \mathrm{~nm}$ to $1.4 \mu \mathrm{m}$, with a clear spectral feature centred at $520 \mathrm{~nm}$, which is referred to the so-called blue-peak [24, 25], as shown in Fig. 1(a). The inset shows a corresponding far field pattern of the visible part of the SC emission (as photographed $20 \mathrm{~cm}$ away from the output face of the YAG crystal), which is dominated by green spectral components, associated with the intense blue peak. With increasing the input pulse energy, no further blue-shifted broadening is recorded, as indicated by a fairly stable cut-off wavelength at $460 \mathrm{~nm}$. However, the blue peak itself experiences a marked broadening up to $700 \mathrm{~nm}$, as shown in Fig. 1(b), also verified by the emergence of strong yellow-red emission in the far field, shown in respective insets. At the same time, there is a slight increase of the red-shifted spectral broadening up to to $1.5 \mu \mathrm{m}$, which is accompanied by a slight deterioration of the far field SC emission pattern, as illustrated in Fig. 11(c).
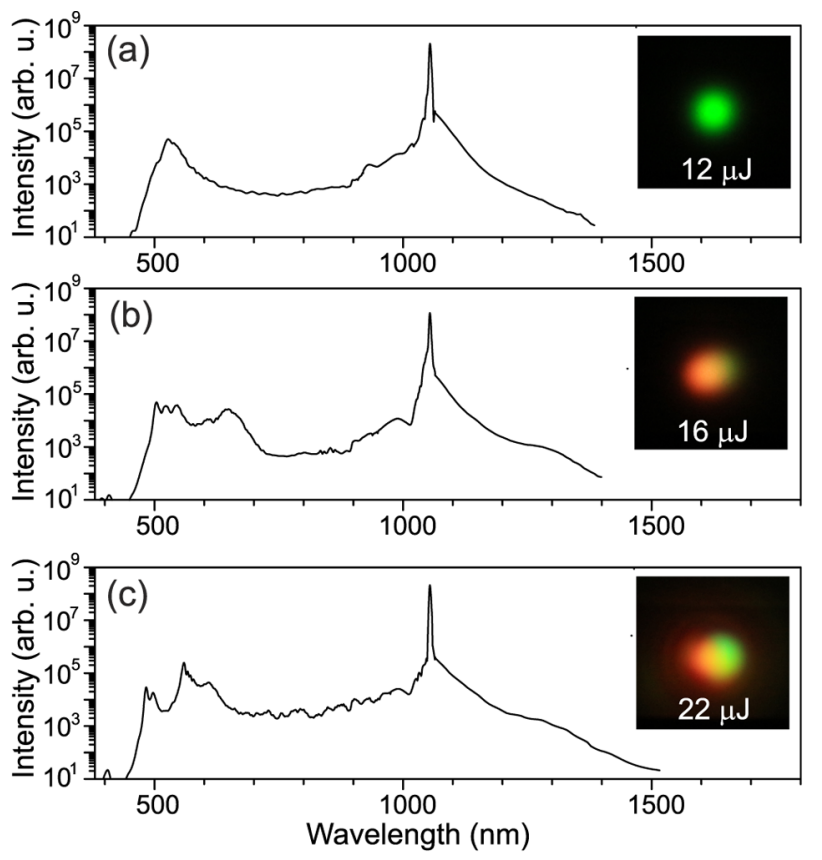

Fig. 1. Supercontinuum spectra generated with $1.1 \mathrm{ps,}$ $1055 \mathrm{~nm}$ laser pulses in the $15 \mathrm{~mm}$-long YAG crystal. Insets show the screenshots of the SC emission patterns in the visible spectral range and indicate the input pulse energy. 
In the sapphire crystal the SC generation is observed at almost twice higher input pulse energies, owing to its smaller nonlinearity and thus larger critical power for self-focusing, see Table. The spectral broadening in the sapphire crystal was detected with the input pulse energy of $20 \mu \mathrm{J}$, that corresponds to $6.3 P_{\text {cr }}$. The SC spectra as measured at the output of the sapphire crystal are presented in Fig. 2 and show the same relevant features as those recorded in YAG. With the input pulse energy of $21 \mu \mathrm{J}$ the SC spectrum spans from $410 \mathrm{~nm}$ to $1.3 \mu \mathrm{m}$, with a distinct blue peak centred at $495 \mathrm{~nm}$, as shown in Fig. 2(a). The short wave cut-off wavelength of the SC spectrum is at $410 \mathrm{~nm}$, which remains constant with further increase of the input pulse energy, while the red-shifted spectral broadening slightly extends up to $1.4 \mu \mathrm{m}$, as shown in Figs. 2(b) and (c). However, with increasing the input pulse energy, the far field patterns of visible SC emission become strongly deteriorated, as shown in the insets of Figs. 2(b) and (c), indicating the onset of multiple filamentation.
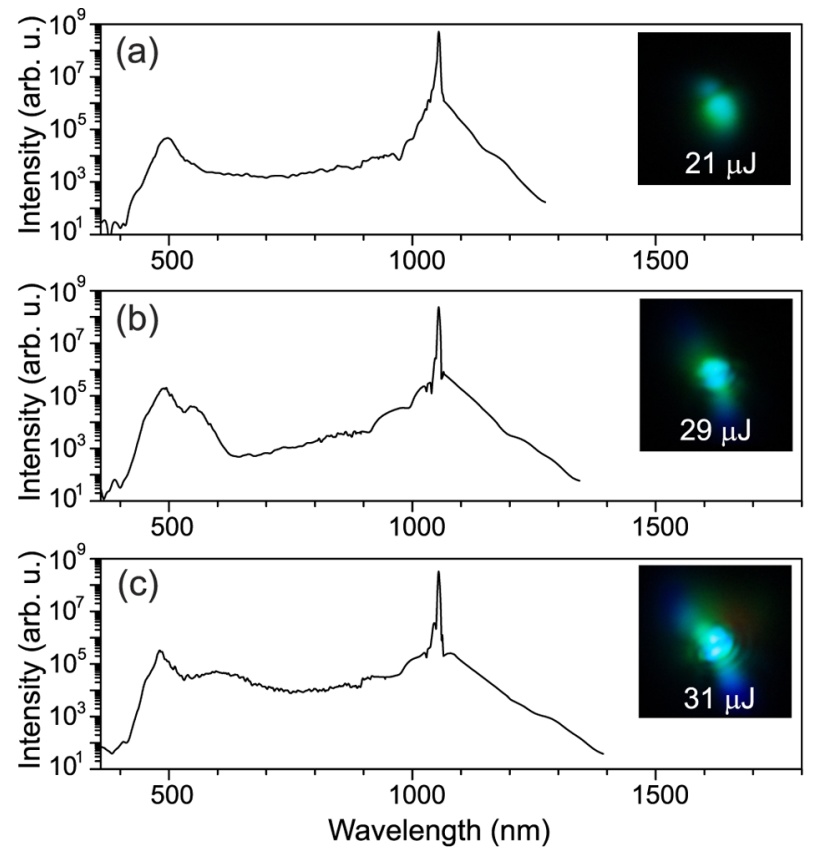

Fig. 2. Supercontinuum spectra generated with $1.1 \mathrm{ps,}$ $1055 \mathrm{~nm}$ laser pulses in the $15 \mathrm{~mm}$-long sapphire crystal. Insets show the screenshots of the SC emission patterns in the visible spectral range and indicate the input pulse energy.

Figure 3 compares the intensity distributions of the input and SC producing output beams. Figure 3 (a) shows the profile of the spatially filtered input Gaussian beam with a $100 \mu \mathrm{m}$ FWHM diameter, as recorded at the input face of the nonlinear medium. Figures $3(\mathrm{~b})$ and $(\mathrm{c})$ present the resulting intensity distributions as recorded at the output face of the YAG crystal with the input energy of $12 \mu \mathrm{J}$ and the sapphire crystal with the input energy of $21 \mu \mathrm{J}$, respectively. In both cases, the recorded intensity distributions attest formation of a narrow single filament with a $\sim 20 \mu \mathrm{m}$ FWHM diameter of the central core, which carries approximately $10 \%$ of the total input energy, as verified by spatial integration of the intensity profiles. A stable single filament formation regime in YAG is maintained within quite broad input pulse energy margins, in the 11-22 $\mu \mathrm{J}$ range, whereas formation of a single filament in sapphire was observed in the slightly reduced, 21-25 $\mu$ input energy range.
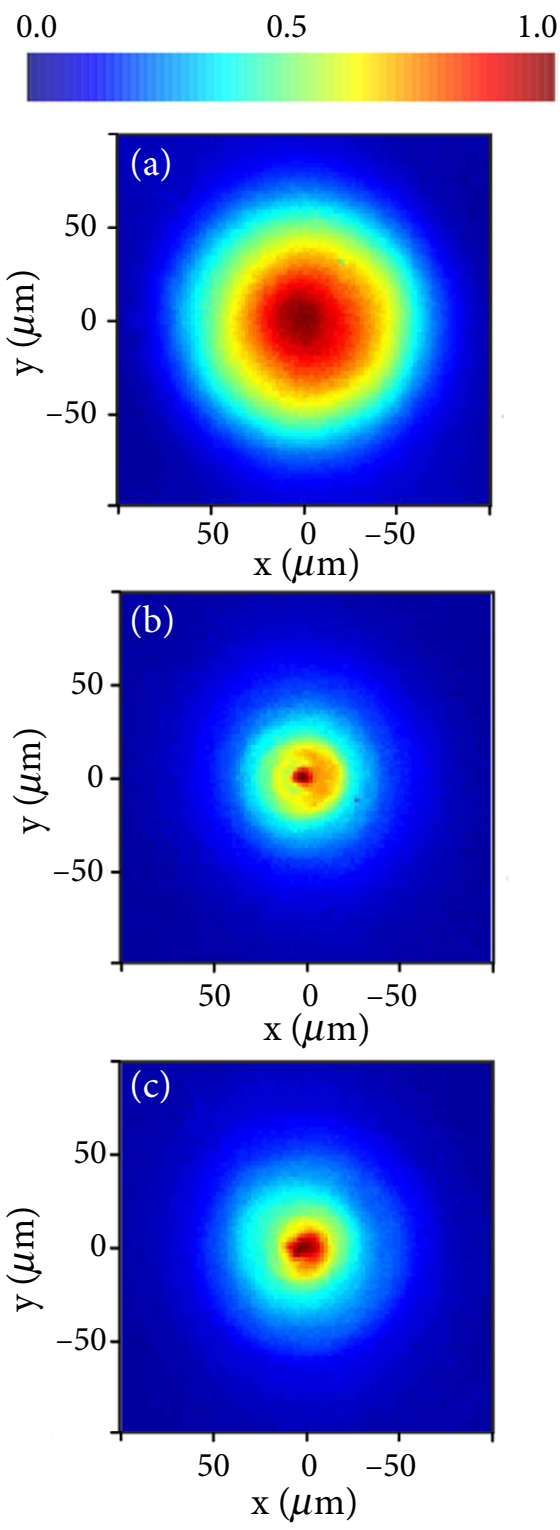

Fig. 3. Normalized spatial intensity profiles of (a) the input beam at the front face of the nonlinear medium, and the single filament at the output face of (b) YAG and (c) sapphire samples, as recorded with the input pulse energies of 12 and $21 \mu \mathrm{J}$, respectively. 
Under these operating conditions, we investigated the statistical properties of the SC spectra, which are of importance as far as applications of the SC emission, e. g. for seeding the optical parametric amplifiers, are considered. The statistical results of picosecond SC in YAG in the 400-950 $\mathrm{nm}$ range are summarized in Fig. 4. Figure 4(a) depicts 2000 consecutive SC spectra (shown by multiple grey curves) as recorded in a single-shot regime with the input pulse energy of $12 \mu \mathrm{J}$ and demonstrates that all SC spectra exhibit well-reproducible shapes and stable spectral intensity. The fluctuations of the SC spectral intensity are expressed by the root-mean-square (RMS) error and are plotted in Fig. 4(b). The SC fluctuations in the wavelength range of 650-950 nm are generally less than $10 \%$ and attest good stability of the SC, as measured with RMS of the input pulse energy fluctuations of $5 \%$. On the other hand, the SC spectral intensity at the vicinity of the blue peak shows markedly increased fluctuations, which are typical statistical features observed in the case of SC generation with femtosecond laser pulses as due to intrinsic intensity and phase noise present in the input pulses, see e. g. []] for details. It has to be mentioned, however, that femtosecond pulses in general produce much more stable SC, whose fluctuations are of the same order as the laser pulse fluctuations themselves [6,8]. Almost identical statistics and wavelength-dependent stability features of the SC were measured in sapphire (not shown), in the 21-25 $\mu \mathrm{J}$ input pulse energy range, before the multiple filamentation occurs.
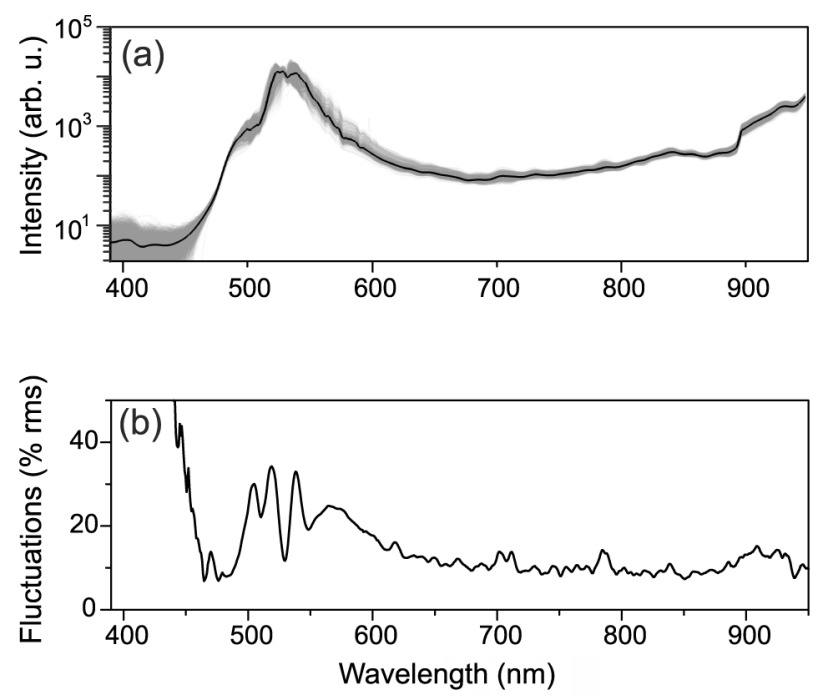

Fig. 4. (a) 2000 consecutive SC spectra generated in YAG with the input pulse energy of $12 \mu \mathrm{J}$. The black curve shows an average spectrum. (b) Fluctuations of the SC spectral intensity. The RMS of the input pulse energy fluctuations is $5 \%$.

\section{Impact of permanent material modification}

The most critical issue in SC generation with picosecond laser pulses concerns permanent modification or even catastrophic optical damage of the solid-state nonlinear medium. In the measurements reported above, no catastrophic optical damage either of the output face or inside the bulk was observed in a single shot regime for the input energy range used in the experiments. The maximum input energy of $31 \mu \mathrm{J}$ corresponds to a fluence of $0.30 \mathrm{~J} / \mathrm{cm}^{2}$, and the estimated fluence in the central core of the filament at the output of the nonlinear medium under conditions of stable SC generation is less than $1 \mathrm{~J} / \mathrm{cm}^{2}$; these values are safely below the threshold values for YAG and sapphire, as provided in Table.

However, we observed that irradiation of the nonlinear medium by a certain number of consecutive laser shots eventually leads to permanent modification of the material, as verified by distortions of the output beam (filament) and the shape of the SC spectrum. For instance, permanent material modification at the filament site starts to manifest itself after approximately 2000 laser shots with the input pulse energy of $14.5 \mu \mathrm{J}$ in YAG and after approximately 18000 laser shots with the input pulse energy of $31 \mu \mathrm{J}$ in sapphire. On the other hand, with lower input energies, $12 \mu \mathrm{J}$ in YAG and $21 \mu \mathrm{J}$ in sapphire, i. e. under operating conditions which produce a single filament and stable SC generation, no permanent modification was detected after the same respective numbers of laser shots.

Although we did not perform detailed monitoring of modification dynamics, which in fact requires complex detection techniques, in what follows we demonstrate how the relevant output parameters (the spatial profile of the beam and the shape of the SC spectrum) become altered in a modified medium.

The beam distortions were examined by performing a simple quantitative test, by launching a low intensity laser beam (i. e. by simply reducing the power of the input beam well below the critical power for self-focusing) through the irradiated site and monitoring its output intensity profile. In an unmodified material, propagation of the input beam is just a subject to diffraction and the beam profile at the output exhibits a smooth, Gaussian-shaped intensity distribution, as shown in Fig. 5(a). In contrast, permanent material modification results in the change of the refractive index at the irradiated site (at the filament core), thus producing an area with the altered refractive index which distorts the low intensity beam, as shown in Fig. 5(b). Figures 5(c) and (d) compare the intensity distributions of the filaments, which are formed in modified YAG and sapphire crystals, revealing clear differences as compared to the spatial intensity distributions of the filaments generated 

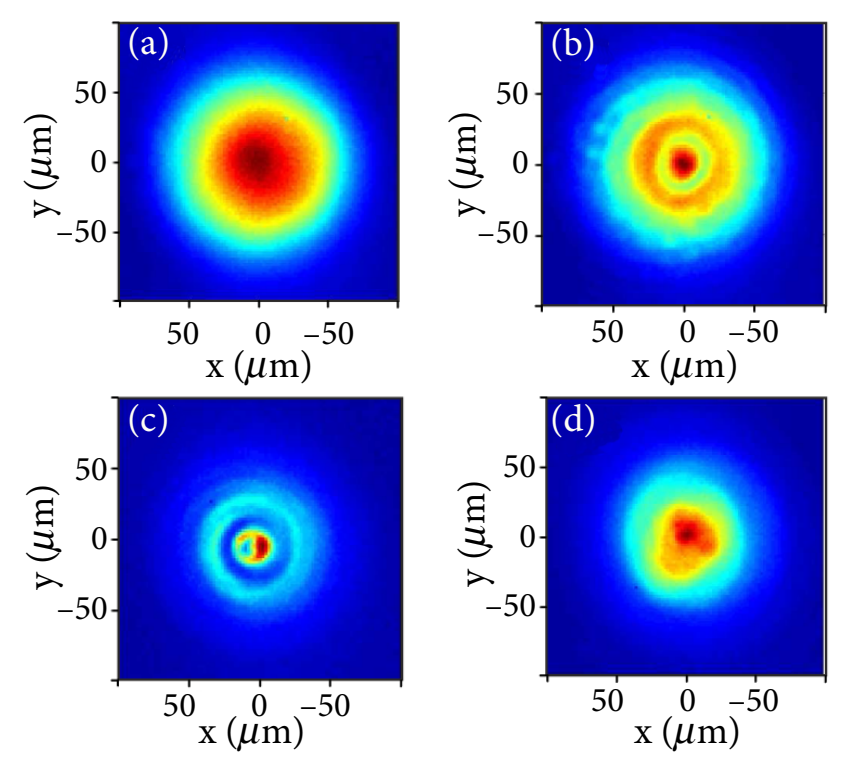

Fig. 5. Spatial profiles of a low energy beam at the output of the YAG crystal: (a) in the absence and (b) in the presence of material modification. Spatial profiles of the filaments generated in (c) modified YAG and (d) modified sapphire. in unmodified media, as shown in Figs. 3(b) and (c), respectively. In particular, in the YAG crystal, the filament is apparently expelled out of the propagation axis, suggesting that the modified area has lower refractive index, which could be attributed to compressional stress induction 26 . In contrast, the modified area in sapphire is not so clearly confined, and we attribute this fact in part to the beam break-up into multiple filaments, whose spatial positions slightly vary from one laser shot to another, resulting in much larger affected volume. On the other hand, we may speculate that the marked differences in exposure time required to permanently modify YAG and sapphire crystals could be related to different dynamics of the electron trapping in the conduction band (e. g. colour centre formation) [28, 29, which in turn induces additional energy bands strongly absorbing in the near infrared. Measurements of the transmitted energy also reveal that in the case of stable SC generation, approximately $15 \%$ of the input energy is nonlinearly absorbed in YAG, while twice smaller amount of energy (7\%) is absorbed in sapphire. Relatively larger nonlinear absorption in YAG is attributed to the lower order of the multiphoton absorption; we estimate six photon absorption in YAG $\left(E_{\mathrm{g}}=6.5 \mathrm{eV}\right)$, and nine photon absorption in sapphire $\left(E_{\mathrm{g}}=9.9 \mathrm{eV}\right)$, with a single photon energy of $1.15 \mathrm{eV}$.

Finally, we verified that filamentation in the modified medium still leads to spectral broadening and SC generation, however, the recorded spectra exhibit distinct differences in the shapes of the SC spectra, as presented in Fig. 6. Interestingly, the material (YAG in the present case) modification has no apparent effect on the position and spectral intensity of the blue peak, while the SC emission in the near-infrared (around $800 \mathrm{~nm}$ ) spectral range becomes strongly, and even completely (at lower input pulse energy) suppressed.
We should mention that experiments of the SC generation in laser-modified fused silica reported a different character of changes of the SC spectra, i. e. showing a continuous spectral shrinkage in the visible range [27]. The observed differences could be attributed to the structural differences of the nonlinear media (amorphous vs crystalline) and possible onset of thermal effects, since the abovementioned experiments were performed at very high $(100 \mathrm{kHz})$ repetition rate.
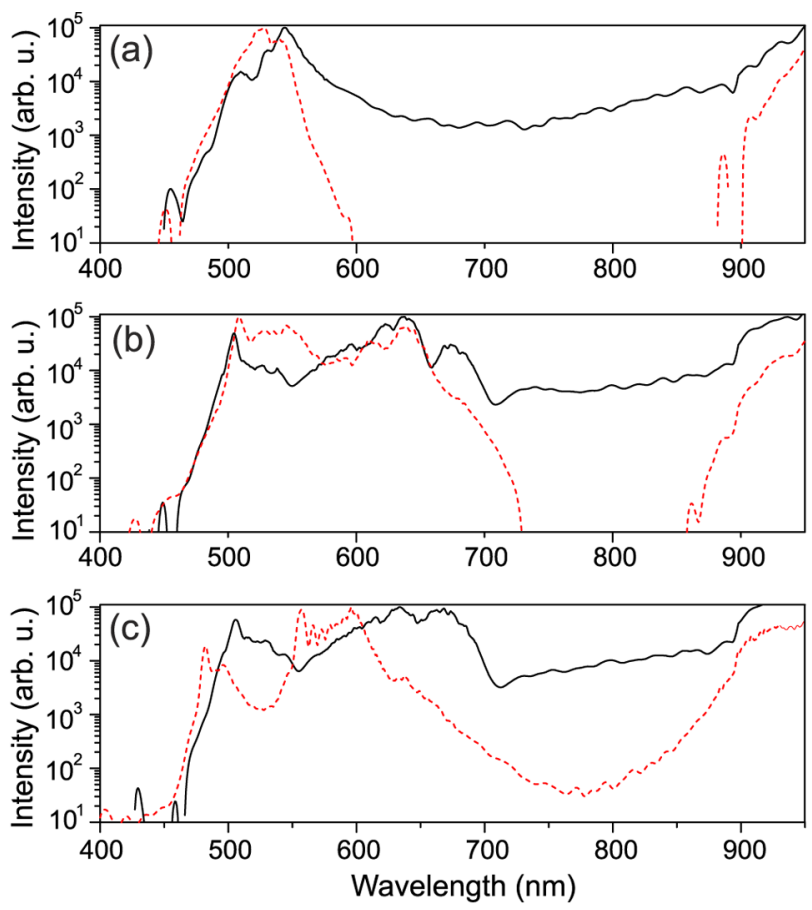

Fig. 6. Comparison of the visible-near infrared SC spectra in YAG in the absence (solid curves) and in the presence (dashed curves) of material modification, as recorded with different input pulse energies: (a) $12 \mu \mathrm{J}$, (b) $16 \mu \mathrm{J}$, (c) $22 \mu \mathrm{J}$. 


\section{Conclusions}

In conclusion, we presented an experimental study of the SC generation in YAG and sapphire crystals with $1.1 \mathrm{ps}, 1055 \mathrm{~nm}$ laser pulses. Stable and reproducible SC spectra spanning from $460 \mathrm{~nm}$ to $1.4 \mu \mathrm{m}$ in YAG and from $410 \mathrm{~nm}$ to $1.3 \mu \mathrm{m}$ in sapphire were generated in a single filament formation regime in loose focusing conditions, without the onset of the optical damage. We identify that permanent material modification that develops after irradiation by several thousands of successive laser shots and results in the change of the local refractive index is the main factor which strongly affects filament formation and alters the SC spectral shapes.

Our results may be relevant concerning the development of simple, efficient and inexpensive tabletop OPCPA-based laser systems, which provide few optical cycle pulses and which are solely based on picosecond laser sources, see e. g. [30]. However, the temporal structure of picosecond supercontinuum, which is of importance for the above applications and whose measurements constitute a nontrivial experimental task, has still to be uncovered in future studies. From a general point of view, it is possible that picosecond filamentation dynamics resulting in spectral superbroadening may appear different as compared to that of femtosecond filamentation, due to the increased role of the free electron plasma generated by longer laser pulses.

\section{Acknowledgements}

This research was funded by the European Social Fund under the Global Grant measure (Grant No. VP1-3.1ŠMM-07-K-03-001).

\section{References}

[1] A. Brodeur and S.L. Chin, Ultrafast white-light continuum generation and self-focusing in transparent condensed media, J. Opt. Soc. Am. B 16, 637-650 (1999).

[2] C. Nagura, A. Suda, H. Kawano, M. Obara, and K. Midorikawa, Generation and characterization of ultrafast white-light continuum in condensed media, Appl. Opt. 41, 3735-3742 (2002).

[3] V.P. Kandidov, O.G. Kosareva, I.S. Golubtsov, W. Liu, A. Becker, N. Akozbek, C.M. Bowden, and S.L. Chin, Self-transformation of a powerful femtosecond laser pulse into a white-light laser pulse in bulk optical media (or supercontinuum generation), Appl. Phys. B 77, 149-165 (2003).

[4] A.K. Dharmadhikari, F.A. Rajgara, and D. Mathur, Systematic study of highly efficient white-light generation in transparent materials using intense femtosecond pulses, Appl. Phys. B 80, 61-66 (2005).

[5] J.B. Ashcom, R.R. Gattass, C.B. Schaffer, and E. Mazur, Numerical aperture dependence of damage and supercontinuum generation from femtosecond laser pulses in bulk fused silica, J. Opt. Soc. Am. B 23, 2317-2322 (2006).

[6] M. Bradler, P. Baum, and E. Riedle, Femtosecond continuum generation in bulk laser host materials with sub- $\mu$ J pump pulses, Appl. Phys. B 97, 561574 (2009).

[7] D. Majus, V. Jukna, E. Pileckis, G. Valiulis, and A. Dubietis, Rogue-wave-like statistics in ultrafast white-light continuum generation in sapphire, Opt. Express 19, 16317-16323 (2011).

[8] D. Majus and A. Dubietis, Statistical properties of ultrafast supercontinuum generated by femtosecond Gaussian and Bessel beams: a comparative study, J. Opt. Soc. Am. B 30, 994-999 (2013).

[9] V. Jukna, J. Galinis, G. Tamošauskas, D. Majus, and A. Dubietis, Infrared extension of femtosecond supercontinuum generated by filamentation in solid-state media, Appl. Phys. B 116, 477-483 (2014).

[10]A. Saliminia, S.L. Chin, and R. Vallée, Ultrabroad and coherent white light generation in silica glass by focused femtosecond pulses at $1.5 \mu \mathrm{m}$, Opt. Express 13, 5731-5738 (2005).

[11]E.O. Smetanina, V.O. Kompanets, S.V. Chekalin, A.E. Dormidonov, and V.P. Kandidov, Anti-Stokes wing of femtosecond laser filament supercontinuum in fused silica, Opt. Lett. 38, 16-18 (2013).

[12]M. Durand, K. Lim, V. Jukna, E. McKee, M. Baudelet, A. Houard, M. Richardson, A. Mysyrowicz, and A. Couairon, Blueshifted continuum peaks from filamentation in the anomalous dispersion regime, Phys. Rev. A 87, 043820 (2013).

[13]J.A. Dharmadhikari, R.A. Deshpande, A. Nath, K. Dota, D. Mathur, and A.K. Dharmadhikari, Effect of group velocity dispersion on supercontinuum generation and filamentation in transparent solids, Appl. Phys. B 117, 471-479 (2014).

[14]F. Silva, D.R. Austin, A. Thai, M. Baudisch, M. Hemmer, D. Faccio, A. Couairon, and J. Biegert, Multi-octave supercontinuum generation from mid-infrared filamentation in a bulk crystal, Nature Commun. 3, 807 (2012).

[15]J. Darginavičius, D. Majus, V. Jukna, N. Garejev, G. Valiulis, A. Couairon, and A. Dubietis, Ultrabroadband supercontinuum and third-harmonic generation in bulk solids with two optical-cycle carrier-envelope phase-stable pulses at $2 \mu \mathrm{m}$, Opt. Express 21, 25210-25220 (2013).

[16]R.R. Alfano, The Supercontinuum Laser Source (Springer, 2006).

[17] M. Bradler and E. Riedle, in: Advanced Solid-State Photonics (ASSP) 2011, Paper AMD4. 
[18] M. Bradler and E. Riedle, in: Proceedings of the Conference on Lasers and Electrooptics (CLEOEurope) 2011, Paper CF3-2.

[19] M. Schulz, R. Riedel, A. Willner, T. Mans, C. Schnitzler, P. Russbueldt, J. Dolkemeyer, E. Seise, T. Gottschall, S. Hädrich, S. Duesterer, H. Schlarb, J. Feldhaus, J. Limpert, B. Faatz, A. Tünnermann, J. Rossbach, M. Drescher, and F. Tavella, Yb:YAG Innoslab amplifier: efficient high repetition rate subpicosecond pumping system for optical parametric chirped pulse amplification, Opt. Lett. 36, 2456-2458 (2011).

[20] R. Riedel, A. Stephanides, M.J. Prandolini, B. Gronloh, B. Jungbluth, T. Mans, and F. Tavella, Power scaling of supercontinuum seeded megahertz-repetition rate optical parametric chirped pulse amplifiers, Opt. Lett. 39, 1422-1424 (2014).

[21]S. Xu, J. Qiu, T. Jia, C. Li, H. Sun, and Z. Xu, Femtosecond laser ablation of crystals $\mathrm{SiO}_{2}$ and YAG, Opt. Commun. 274, 163-166 (2007).

[22] O. Uteza, B. Bussière, J.-P. Chambaret, P. Delaporte, T. Itina, and M. Sentis, Laser-induced damage threshold of sapphire in nanosecond, picosecond and femtosecond regimes, Appl. Surf. Sci. 254, 799-803 (2007).

[23] M.J. Weber, Handbook of Optical Materials (CRC, 2003).

[24]D. Faccio, A. Averchi, A. Couairon, A. Dubietis, R. Piskarskas, A. Matijosius, F. Bragheri, M.A. Porras, A. Piskarskas, and P. Di Trapani, Competition between phase matching and stationarity in Kerrdriven optical pulse filamentation, Phys. Rev. E 74, 047603 (2006).

[25]D. Faccio, A. Averchi, A. Lotti, M. Kolesik, J.V. Moloney, A. Couairon, and P. Di Trapani, Generation and control of extreme blueshifted continuum peaks in optical Kerr media, Phys. Rev. A 78, 033825 (2008).

[26] S. Xu, J. Qiu, C. Li, H. Sun, and A. Xu, Direct writing waveguides inside YAG crystal by femtosecond laser, Opt. Commun. 282, 4810-4814 (2009).

[27]D. Paipulas, A. Balskienè, and V. Sirutkaitis, Experimental study of filamentation and supercontinuum generation in laser-modified fused silica, Lith. J. Phys. 52, 327-333 (2012).

[28] S. Guizard, P. Martin, Ph. Daguzan, G. Petite, P. Audebert, J.P. Geindre, A. Dos Santos, and A. Antonetti, Contrasted behavior of an electrongas in $\mathrm{MgO}, \mathrm{Al}_{2} \mathrm{O}_{3}$ and $\mathrm{SiO}_{2}$, Europhys. Lett. 29, 401-406 (1995).

[29] P. Martin, S. Guizard, Ph. Daguzan, G. Petite, P. D'Oliveira, P. Meynadier, and M. Perdrix, Subpicosecond study of carrier trapping dynamics in wide-band-gap crystals, Phys. Rev. B 55, 57995810 (1997).

[30] A. Vaupel, N. Bodner, B. Webb, L. Shah, and M. Richardson, Concepts, performance review, and prospects of table-top, few-cycle optical parametric chirped-pulse amplification, Opt. Eng. 53, 051507 (2014).

\title{
SUPERKONTINUUMO GENERAVIMAS YAG IR SAFYRO KRISTALUOSE ŽADINANT PIKOSEKUNDINIAIS LAZERIO IMPULSAIS
}

\author{
I. Gražulevičiūtė, M. Skeivytė, E. Keblytė, J. Galinis, G. Tamošauskas, A. Dubietis \\ Vilniaus universiteto Kvantines elektronikos katedra, Vilnius, Lietuva
}

\section{Santrauka}

Eksperimentiškai ištirtas superkontinuumo generavimas YAG ir safyro kristaluose žadinant 1,1 ps trukmès, $1055 \mathrm{~nm}$ bangos ilgio lazerio impulsais. Švelnaus fokusavimo sąlygomis pradinio impulso galių intervale nuo 6 iki $10 P_{\mathrm{cr}}$ sužadinta stabili šviesos gija, kuri lèmé gerai atsikartojančio superkontinuumo generavimą ir spektro plètrą atitinkamai nuo $460 \mathrm{~nm}$ iki $1,4 \mu \mathrm{m}$ YAG ir nuo $410 \mathrm{~nm}$ iki 1,3 $\mu \mathrm{m}$ safyro kristaluose. Nustatyta, kad ilgalaike medžiagos savybių modifikacija dèl daugelio pasikartojančiu impulsų poveikio yra lemiamas veiksnys, iškraipantis šviesos gijos intensyvumo skirstinị ir labai modifikuojantis superkontinuumo spektrą. 